\title{
PARTICIPACIÓN DEL VARÓN EN LA SOLICITUD DE LOS SERVICIOS DE SALUD REPRODUCTIVA EN LA SIERRA CENTRAL DEL PERÚ*
}

\author{
Romero Santillana, María Leonor ${ }^{1}$ y Porras Iparraguirre, Ruth Mónica² \\ Facultad de Enfermería de la Universidad Nacional del Centro del Perú
}

\begin{abstract}
RESUMEN
La información respecto a las bondades de los servicios de salud reproductiva, es orientada a la población femenina, dejando de lado al gran actor, quien toma las decisiones más importantes, girando en función de él los aspectos sociales, económicos y reproductivos de la familia; por lo que se estudió la participación del varón y su influencia en la solicitud de atención de los servicios de salud reproductiva realizando un estudio descriptivo, no experimental de corte transversal en el distrito 9 de Julio de la provincia de Concepción, 2003. Se concluyó: $80 \%$ de la población de mujeres encuestada y en unión estable con su pareja solicitó alguna vez atención en los servicios de salud reproductiva (control prenatal, planificación familiar, control de cáncer ginecológico, atención en gineco-obstetricia). Las razones por las que el varón influye positivamente en la solicitud de atención de los servicios de salud reproductiva son:
\end{abstract}

- Mayor nivel de instrucción del varón.

- Sexo femenino del proveedor del servicio de salud reproductiva.

- Percepción favorable de la calidad de atención en los servicios salud reproductiva.

- Mayor conocimiento de los signos de alarma.

- Percepción de la importancia de la atención en los servicio de salud reproductiva.

Existe una relación estadísticamente significativa entre la solicitud de los servicios de salud reproductiva y que el varón perciba como importante los SSR, lo cual determina su participación activa en el proceso de atención en todos y cada uno de los servicios involucrados en la atención de salud reproductiva.

Palabras clave: salud, reproductiva, control prenatal, planificación, familiar.

\section{MAN PARTICIPATION IN REQUESTING THE VARN IN THE REQUEST OF THE SERVICES OF REPRODUCTIVE HEALTH IN THE CENTRAL REGION OF THE PERU}

\begin{abstract}
The information regarding the effectiveness in the services of reproductive health, is directed to the decisions hanng arou d him the feminine population, leaving aside the great actor who takes acording the most important, the function of the social, economic and reproductive aspects of the family. For what was studied the male's participation and their influence in the application of attention of the services of reproductive health from this as descriptive study, of not experimental of transverse new was realized in the districtor nine of Julio of Concepcion's County, 2003 we concluded: the interviewed population's of women $80 \%$ and in stable union with their couple requested attention at some time in the services of reproductive health (Prenatal Control, family planning, control of gynecological cancer, attention in gineco-obstetrics).

The reasons for those that the male influences positively in the application of attention of the services of reproductive health are:

Higher level of the male's instruction.

The supplier's of the service feminine sex of MR.

Favorable perception of the quality of attention in the services SR.
\end{abstract}

\footnotetext{
* Este trabajo de investigación fue recibido el 20/04/2004 retornado para su revisión 20/06/2007 y aprobado para su publicación 10/12/2007

1. Email.mariaromero @hotmail.com

2. Email:ipomoru@hotmail.com
} 
Better knowledge of the alarm signs.

Perception fo the importance of the attention in the SSR.

A statistically significant relationship exists among the application of the services of reproductive health and that the male perceives as important the SSR, which determines his active participation in the process of attention in all and each one of the services involved in the attention of reproductive health.

Key Words: Reproductive, health, prenatal control, planning, family.

\section{INTRODUCCIÓN}

La salud reproductiva y perinatal en América Latina no ha mejorado sustancialmente en los últimos 5 años. El análisis epidemiológico del estado de salud de la población de las Américas revela que los grupos más vulnerables continúan siendo las mujeres, especialmente en edad fértil. En un análisis reciente, llevado a cabo por el Banco Mundial, se llegó a la conclusión de que la atención en los servicios de salud reproductiva se cuentan entre las intervenciones más eficaces en función del costo con las cuáles los gobiernos pueden mejorar la salud de los adultos y los niños. Estudios anteriores han demostrado que las mujeres del área rural tienen una probabilidad dos veces mayor de morir por causas maternas que las mujeres del área urbana: 51 versus 24 muertes por cada 100 mil mujeres, respectivamente (Solís, 1994).

Se ha visto también que el varón desempeña un papel importante dentro de la salud reproductiva. Estudios realizados en contextos culturales semejantes (Zonas rurales y semirurales) demuestran que los hombres tienen cierto dominio sobre las mujeres y el poder de las decisiones en el campo de la salud reproductiva. Aspecto que nos motivó a realizar el presente trabajo en el distrito 9 de Julio, de la provincia de Concepción, en el departamento de Junín, sierra central del Perú.

La finalidad de esta investigación fue establecer la participación del varón en la solicitud de atención de los servicios de salud reproductiva en la sierra central del Perú. Para lo que se plantearon los siguientes objetivos: analizar la participación del varón y su influencia en la solicitud de atención de los servicios de salud reproductiva en los puestos de salud de la jurisdicción del centro de salud de Concepción. Teniendo como objetivos específicos: Identificar la proporción de mujeres que cuenta con el apoyo de su pareja y solicita atención en salud reproductiva, Analizar las razones por que los varones influyen positiva 0 negativamente en la solicitud de atención de los servicios de salud reproductiva. Establecer la relación existente entre participación de la pareja masculina y solicitud de atención de los servicios de salud reproductiva

\section{MATERIAL Y MÉTODOS}

El presente trabajo es un estudio descriptivo, no experimental de corte transversal, se realizó en el distrito de 9 de Julio de la provincia de Concepción, en el período 2003. La población estuvo conformada por las parejas del distrito de 9 de Julio, se consideró el $100 \%$ de parejas que cumplieran con los criterios de inclusión. La unidad de análisis fueron las parejas con hijos menores de un año. Como marco muestral se tomó la relación de nombres de niños menores de un año, del archivo del programa de inmunizaciones del establecimiento de salud.

Teniendo en cuenta la naturaleza descriptiva del estudio y buscando lograr una adecuada aproximación para la caracterización sociocultural y económica, se obtuvo los datos de fuentes primarias, para lo cual se utilizó la técnica de entrevista personal, y como instrumento de recolección de datos, un cuestionario estructurado que se aplicó a la unidad de análisis.

Los criterios de inclusión fueron: hogar en el que exista una madre de un niño menor de un año (este último vivo 0 muerto) y que viva en unión estable con su pareja, hogares en los que además de cumplir el criterio anterior, la pareja haya permanecido en el hogar durante el periodo de embarazo, parejas que cumplieron los criterios anteriores y que ambos se encontraban en la zona en el momento de las entrevistas, parejas masculina que estuvieron en completo uso de sus facultades.

Las variables de estudio fueron: solicitud de atención de los servicios de salud reproductiva, apoyo del esposo para la solicitud de atención en salud reproductiva.

El cuestionario fue validado mediante un estudio piloto realizado con 12 padres de hijos menores de un año, en 3 lugares con características similares a los lugares de estudio. Además el instrumento fue sometido a evaluación a través de la opinión de nueve expertos a quienes se entregaron 2 documentos, y un segundo formulario para la evaluación de los expertos. 
Se realizó una limpieza de datos y procedió a la respectiva digitación de las mismas en el programa de SPSS donde se realizó el análisis estadístico respectivo, los cuadros y gráficos fueron elaborados en el programa de Microsoft Excel.

\section{Del estudio cualitativo}

El primer contacto se realizó con los responsables del puesto de salud de 9 de Julio - Concepción, iniciando con un acercamiento y presentación de los objetivos del estudio y posteriormente con algunos de los pobladores que nos ayudaron a contactarnos con el grupo de personas que eran elegibles para el estudio.

Se mostró en todo momento el respeto requerido hacia las costumbres y necesidades de la población, por ejemplo se realizó una reunión par establecer la fecha, hora, lugar en el que se realizarían las dinámicas y/o entrevistas y recolección de datos.

Se desarrollaron guías de preguntas tanto para los grupos focales de varones como para el de las mujeres.

Grupos Focales: se condujeron con grupos de ocho varones y ocho mujeres por separado con esta técnica se logró conocer los contextos en los que se desarrollan las relaciones que se están investigando, así como las percepciones de los servicios de salud reproductiva. La guía de preguntas fue adecuada y probada con grupos de varones urbano - marginales de Huancayo. Cada grupo focal tuvo aproximadamente una duración de dos horas, fueron totalmente grabadas previa autorización de los participantes

La información fue codificada e ingresada al ATLAS - TI, el análisis se realizó utilizando los testimonios recogidos en los grupos focales.

\section{RESULTADOS}

En nuestro estudio el total de parejas encuestadas es de 56 , las cuales permanecían en unión estable y tenían un niño menor de un año, todas ellas procedentes del distrito de 9 de Julio. La mediana de edad de varones encuestados es de 30 a 34 años de edad, siendo la moda de 25 a 29 años con un $28,57 \%$.

En las mujeres encuestadas la mediana de edad es de 25 a 29 años, mientras que la moda es de 20 a 24 años de edad $(33,93 \%)$. Se observa que las mujeres tienen aproximadamente cuatro años menos que sus parejas.

Los varones encuestados tienen en mayor proporción secundaria con el $51,79 \%$, y superior con el $17,86 \%$.
Observándose que en el grupo de mujeres existe una mayor proporción de las mismas que es analfabeta $10,71 \%$, y en términos generales las mujeres en aproximadamente $48 \%$ solo alcanzan primaria o son analfabetas. Esto parece ser debido a la creencia de que el hombre debe estar más preparado para enfrentar una vida en el ámbito público y así poder enfrentar cualquier circunstancia que se le presente, mientras la mujer se ve relegada al ámbito doméstico. Predominantemente los varones prefieren que el proveedor de los servicios de salud reproductiva sea una mujer con el $98,21 \%$.

Tabla 1.- Prefiere mujer como proveedora de los servicios

\begin{tabular}{ccc}
\hline Prefiere mujer & Frecuencia & porcentaje \\
\hline No & 1 & $1,79 \%$ \\
si & 55 & $98,21 \%$ \\
Total & 56 & $100 \%$ \\
\hline
\end{tabular}

El hecho de que el proveedor de los servicios sea varón constituye una barrera para apoyar la solicitud de atención en los servicios de SR en el $64,29 \%$ de los varones.

En esta investigación se encontró que aproximadamente el $52 \%$ de los varones opina que las tarifas de atención en el puesto de salud es entre barato y adecuado. La mayor parte de la población que acude a los servicios de SR en esa zona se encuentra satisfecha con el costo del servicio.

En cuanto al tiempo de espera el mayor porcentaje de mujeres esperó menos de una hora $51,79 \%$. Existe un $14,29 \%$ de mujeres que tuvo que esperar mas de 2 horas, aspecto que expresa ligero malestar en las usuarias.

Aunque el $62,50 \%$ de los varones opina que la atención de los proveedores del servicio de salud reproductiva es buena se tiene un $17,86 \%$ que opina que la atención es regular a mala, cifra que debe tenerse en cuenta para el planteamiento de estrategias.

Respecto a la infraestructura, equipo y materiales en los servicios de SR, el $44,65 \%$ de los varones opinan que son regulares a malos aspectos que debe llamar la atención de nuestras autoridades para priorizar la adecuada ambientación de los consultorios destinados a la atención de SR.

En general podríamos decir que el mayor porcentaje de varones que acompañó a su esposa a solicitar atención en los servicios de SR se encuentra satisfecho de la atención que allí se ofrecen. Sin embargo, se tiene un $44,65 \%$ de varones que opina que el lugar donde se brinda la atención en SR es inadecuado razón por la que podría oponerse a que su esposa acuda a dicho establecimiento, motivo por la que se deben implementar mejoras que contribuyan a propiciar el uso de estos servicios. 
Tabla 2.- Como son los servicios de CPN

\begin{tabular}{lcc}
\hline & Frecuencia & Porcentaje \\
\hline No & & \\
corresponde & 19 & 33,93 \\
Buenos & 18 & 32,14 \\
regulares/malos & 19 & 33,93 \\
Total & 56 & 100,00 \\
\hline
\end{tabular}

Las razones que expresan los participantes son: que no existe privacidad, no se puede proteger el pudor de la usuaria, que los equipos no se encuentran en buenas condiciones, y que el ambiente requiere de arreglo en las paredes y mayor ventilación.

Sin embargo, cuando el hombre apoya a su esposa para que ésta acuda a solicitar atención en salud reproductiva es más probable que la mujer solicite dicha atención. En nuestro estudio se encontró que cuando el esposo apoya que la mujer acuda a los servicio de salud reproductiva el $80,49 \%$ de ellas lo hace. Por otro lado si el varón considera que es importante el servicio de salud reproductiva entonces las mujeres solicitan en mayor proporción $84,62 \%$ esta atención, mientras que cuando el varón no considera importante el servicio de salud reproductiva, sólo el $25 \%$ de las mujeres solicita esta atención, relación que es estadísticamente significativa y que comprueba nuestra hipótesis.

Tabla 3.- Número de veces que acude a CPN según importancia del CPN

\begin{tabular}{lrrrr}
\hline \multirow{2}{*}{$\begin{array}{l}\text { Número de } \\
\text { Veces que }\end{array}$} & \multicolumn{4}{c}{ Importancia del CPN } \\
\cline { 2 - 5 } acude a CPN & \multicolumn{2}{c}{ si } & \multicolumn{3}{c}{ no } \\
\cline { 2 - 5 } $\mathbf{N}^{\mathbf{0}}$ & $\%$ & $\mathbf{N}^{0}$ & $\%$ \\
\hline sin control & 8 & 15,38 & 3 & 75,00 \\
4 a mas & 9 & 17,31 & & \\
1 a 3 & 35 & 67,31 & 1 & 25,00 \\
Total & 52 & 100,00 & 4 & 100,00 \\
Pearson Chi - Square: & 0,01 & & \\
\hline
\end{tabular}

\section{DISCUSIÓN}

En Junín y en todo el Perú se ha venido priorizando la atención en salud de la mujer y el niño, esto debido a las funestas consecuencias familiares, sociales y económicas que trae consigo la muerte de una madre, y por otro lado a que los niños son el potencial social, cultural y económico de nuestro país.

Es también una realidad que los programas de información, educación y comunicación orientados a elevar las coberturas de atención en los servicios de Salud Reproductiva (SR), están dirigidos prioritariamente a la mujer, siendo los hombres los grandes olvidados en este tema, como si no tuvieran derecho u obligación a compartir sus sentimientos y necesidades en esta esfera.
Según los analistas dualistas, la herencia colonial y patriarcal nos legó un sistema genérico en el cual las categorías masculina y femenina se organizaban en esferas netamente separadas y mutuamente complementarias.

"La mujer en la casa y el hombre en la calle" la mujer es la "reina del hogar" y el hombre su opuesto complementario, debía proteger del mundo exterior el "sagrado santuario de la familia" y proveer su sustento. Las esferas política y económica (en lo que se refiere a relaciones con el mundo exterior) eran su feudo y responsabilidad, de allí que reclamase la autoridad sobre el conjunto familiar".

"Ser hombre adulto significa entonces, ejercer los pequeños poderes domésticos y familiares. Tener sobre quién ejercer el poder, es un hecho fundamental para la masculinidad. Ser padre significa en el mundo patriarcal ser tutor de otros. En general son tutores de la cónyuge y de sus hijos pero también ser tutores de quien se deje. Los hombres tutoran como las mujeres maternizamos, o como conducimos nuestro proceso reproductivo, incluso en la vida sexual cotidiana, donde muchas veces la mujer se ve obligada a aceptar un coito desagradable, o que sabe la pone en riesgo de contraer un infección vaginal o peor aún un embarazo no deseado."

En la actualidad diferentes estudios muestran el importante rol que le toca desempeñar al hombre como pareja, especialmente en lo relacionado a la salud sexual y reproductiva, por lo tanto es importante saber: cómo es que el hombre participa en las decisiones relacionadas con la salud reproductiva (salud reproductiva) de la mujer; que conlleva a que esta mujer solicite o no atención en los servicios de salud reproductiva, y qué aspectos pueden influir positiva o negativamente en la participación masculina.

En nuestro estudio a través de los grupos focales se pudo evidenciar que el hombre se siente mejor preparado por tener más fuerza física, lo cual está asociada directamente a su nivel de inteligencia, por lo tanto la mujer que es más débil obviamente será menos inteligente a continuación una cita de unvarón:

"...cuando los esposos son celosos, que vas a ir a atenderte, se molestan, creen que algo tenemos con el Dr. de la posta, tonteras piensan..." (Grupo Focal Mujeres - Distrito 9 de Julio).

Da miedo ir porque no sabes que te van hacer cuando te controlan, a una señora que fue a su control le cortaron un pedazo de su parto para el "papanicolon" (papanicolaou), diciendo...". (Grupo Focal Mujeres - Distrito 9 de Julio)

"Siempre el hombre es más juerte, inteligente, trabaja, consigue la plata, soluciona los problemas, mas bien la mujer es débil, ella tiene que estar en la casa, cuidar a los 
hijos, animales, se tiene problema... para eso está su esposo para solucionar" (Grupo Focal, Varones 9 de Julio).

Las razones que expresan tantos varones y mujeres son:

- El temor a lo desconocido, algunas de las participantes, refieren temor a los procesos a desarrollarse durante la atención, le temen a los exámenes internos tacto, etc. Es importante para ellas saber en que consiste la atención de control prenatal, debido a que algunos miembros de la comunidad comentan falsos procedimientos que asustan a aquellas mujeres que nunca acudieron a un establecimiento de salud.

- Vergüenza, al sentirse invadidas en su intimidad, exponer su cuerpo y genitales.

- Que el esposo no permita que acuda, por factores relacionados al sexo del proveedor aspecto que tiene que ver con la hombría del esposo quien se sentiría disminuido si otro hombre "toca" a su esposa, se tiene una percepción no profesional del proveedor masculino.

Un factor importante para ellas es el apoyo del esposo, y con quien dejar a los niños.

"Mejor es que sea una mujer que le atienda a mi señora, señorita, cuando es hombre a veces se preocupa en mirar otras cosas, ... hasta tus vecinos se ríen de ti...encima, el Dr. Por estar mirando ni les atiende bien, las señoritas son mas pacienzudas, con mas cariño parece le atiende" (Grupo Focal, Varones 9 de Julio).

"Tu siendo mujer casada, tenes que fijarte que a tu esposo no le molesta que te hagues mirar tu parto. o tu barriga, mejor es con su consentimiento que te vayes hacer ver, porque ellos piensan mal, para hacerte manosear con ese doctor seguro estas yendo dicen... por eso yo mejor me voy a otra posta en que atiende la señorita, si no, voy mirando y si hay una señorita me hago ver si no no..." (Grupo Focal, Mujeres 9 de Julio).

El sexo del proveedor del servicio de SR es importante para el acceso a estos servicios. Es unánime la opinión de las participantes de que la atención en salud reproductiva debe ser realizada por una mujer. En la población de estudio el sexo masculino del proveedor constituye una barrera para la solicitud de atención en salud reproductiva. Debido al pudor que ellas manifiestan sentir al tener que mostrar parte de su cuerpo y genitales en los procesos de dicha atención. Por otro lado si ellas aceptan ser atendidas por un proveedor de sexo masculino serán sometidas a las críticas de la suegra además de la desconfianza del esposo. Constituyéndose esta solicitud de atención en motivo de discusión y muchas veces agresión de la pareja hacia la mujer.

"A mí me da vergüenza que un varón me mira mi barriga, ... mis partes, ... por eso, no me he ido hacer ver, en mi gestación, mi esposo claro dice haste ver que importa, pero yo no me he ido señorita". (grupo focal - Distrito 9 de JulioConcepción).

"Siempre el hombre te hace problema, aunque de boca pa' fuera te digue que si, que vayes hacerte ver ni aunque sea con el doctor,... después por las puras te molesta, te fastidia, hasta te pega,... mejor no hacerle caso y no ir, sino buscar a las doctoras nomás". (Grupo Focal Mujeres - Distrito 9 de Julio-Concepción).

Sabemos que la demanda de los servicios de salud depende entre otras cosas de la percepción de la calidad de los servicios de salud así, es importante considerar cual es la percepción de los servicios de salud reproductiva, en el distrito de 9 de Julio.

Las doctoritas te atienden bien de eso no nos quejamos, rápido nomas nos atienden a cualquier hora, un poco el problema es que no tiene su material completo a veces le falta cosas, a veces hasta pegando con esparadapo arregla su aparato... las paredes también están todo despintado chiquito es el local....cuando te esta atendiendo también por la ventana los chiuchis te están mirando...." (Grupo Focal Mujeres - Distrito 9 de Julio-Concepción).

En general podríamos decir que el mayor porcentaje de varones que acompañó a su esposa a solicitar atención en los servicios de SR se encuentra satisfecho de la atención que allí se ofrece. Sin embargo se tiene un $44,65 \%$ de varones que opina que el lugar donde se brinda la atención en SR es inadecuado razón por la que podría oponerse a que su esposa acuda a dicho establecimiento, motivo por el que se deben implementar mejoras que contribuyan a propiciar el uso de estos servicios.

Según ENDES una de las razones por las que las mujeres no se realizan el control prenatal es el desconocimiento de los signos de alarma de la gestación, parto y puerperio.

Es importante resaltar que peligrosamente los varones entrevistados no creen que puedan presentarse complicaciones durante la etapa reproductiva de la mujer. Este hecho nos permite discernir que al desconocer los signos de alarma hace que apoyen poco o se nieguen a que sus esposas acudan a SR, en resumen el $82,14 \%$ de los varones encuestados tienen conocimiento deficiente de los signos de alarma de la salud reproductiva.

"La regla porque es mala sangre lo botan... allí la mujer desfoga para no querer tener las relaciones sexuales" (Grupo Focal Varones - Distrito 9 de Julio-Concepción). 
“... Cuando la mujer hace las relaciones sexuales a cada rato, cuando su esposo le exige tener con su regla le da la cáncer a la matriz" (Grupo Focal Varones - Distrito 9 de Julio-Concepción).

El desconocimiento de los signos de alarma y el no acudir a control prenatal, condiciona que estas mujeres se encuentren en mayor riesgo y lo que es peor no puedan actuar adecuadamente frente a una situación de emergencia o alarma, en el estudio se encontró que aunque el $87,5 \%$ de los varones encuestados tendría una actitud adecuada frente a las situaciones de emergencia gineco obstétrica, se tiene que el $12,50 \%$ de los varones tendría una actitud inadecuada, lo que aumenta el riesgo de sus parejas.

Teniendo como referencia este análisis cuantitativo podríamos asegurar que el mayor nivel de educación que tienen los varones puede condicionar a que estén mejor preparados e informados para enfrentar estas emergencias, sin embargo es delicada la situación respecto al grupo de hombres que tienen conducta inadecuada pues pone en grave peligro de enfermar o morir a la mujer.

El 92,86\% de los varones encuestados considera que la atención en los servicios de salud reproductiva es importante, mientras que el $7,14 \%$ considera que no lo es. En la conversación se pudo determinar que efectivamente gran parte de los varones considera necesario que su esposa acuda a los servicios de SR, identificando como necesidades las infecciones vaginales en primer lugar, el uso de métodos anticonceptivos, el control prenatal, ninguno mencionó la necesidad de despistaje de cáncer ginecológico.

Sin embargo esta percepción de necesidad se encuentra controvertida con ideas erróneas, respecto a la manera de hacer el diagnóstico y/o tratamiento de los problemas identificados, lo que de alguna manera causa temor y hace que éste se oponga a que su esposa solicite atención. En el estudio se encontró que aunque el $73,21 \%$ de los varones apoya a su esposa para que esta acuda a los servicio de salud reproductiva se observa que el $26,79 \%$ no lo hace.

"Sí claro es importante que vaya a hacerse ver cuando le viene la flor blanca, pero solo le deben dar que pastilla es bueno para que se cure, y no cortarle el parto o poner su aparato que le lastima y sangre le saca dice para ver la infección" (Grupo Focal Varones - Distrito 9 de JulioConcepción).

“... es a veces peligroso, por ejemplo su esposa de mi pata se puso la " $T$ " de cobre sin que sepa mi pata, después tuvo su bebe y salió con la "T" en su cabeza puesto como cacho..." (Grupo Focal Varones - Distrito 9 de JulioConcepción).
De acuerdo a las versiones de los hombres a través de los grupos focales se ha podido observar que más que participación en la solicitud de atención de los servicios de salud reproductiva, el varón toma una actitud de controlador, pues son ellos los que al final toman la decisión de tener hijos o no tenerlos, de usar un método anticonceptivo o no y sobre despistaje de cáncer tienen muy pocos 0 nulo conocimiento.

De los grupos focales se pudo discernir que el hombre apoya que la mujer acuda a los servicios de planificación familiar tomando en cuenta prioritariamente el aspecto económico, cuanta mano de obra requieren para el trabajo en la casa (apoyo en la chacra, apoyo para lavar la ropa y/o cocinar). El número de hijos a tener depende de la capacidad proveedora del esposo si mejora su situación económica podría cambiar de opinión y desear tener más hijos, en ningún momento consideran el riesgo que implica para la mujer el tener más hijos.

\section{CONCLUSIONES}

- $\quad$ El $80 \%$ de la población de mujeres encuestada y en unión estable con su pareja solicitó alguna vez atención en los servicios de salud reproductiva (control prenatal, planificación familiar, control de cáncer ginecológico, atención en gineco-obstetricia).

- Las razones por las que el varón influye positivamente en la solicitud de atención de los servicios de salud reproductiva son:

- El mayor nivel de instrucción que alcance el varón.

- El sexo femenino del proveedor del servicio de salud reproductiva.

- La percepción favorable de la calidad de atención en los servicios SR.

- El mayor conocimiento de los signos de alarma.

- La percepción de la importancia de la atención en los servicio de salud reproductiva.

- Existe una relación estadísticamente significativa entre la solicitud de los servicios de salud reproductiva y que el varón perciba como importante los servicio de salud reproductiva, lo cual determina su participación activa en el proceso de atención en todos y cada uno de los servicios involucrados en la atención de salud reproductiva.

\section{LITERATURA CITADA}

Atkin, L. C., Ehrenfeld, N. 1994. Sexualidad y fecundidad adolescente. México.

Atkin, L. C. 1989. El embarazo en la adolescencia en América Latina y El Caribe: causas y consecuencias psicosociales. México. 
Bachmann, C. A. 2004. Riesgo reproductivo. Consejo Nacional de Ciencia y Tecnología. Lima.

Cáceres, C. y col. 1998. Salud sexual en una ciudad joven. Un programa comunitario en salud sexual con y para los jóvenes. IEPO UPCH. REDESS Jóvenes. Lima.

Chirinos, J. 1996. Nivel de conocimientos, actitudes y prácticas asociadas al comportamiento sexual de riesgo en escolares adolescentes de dos colegios de la USE 06, Lima-Norte Perú.

Climent, G., Arias, D. 1996. Reflexiones acerca de las imágenes de género y la maternidad adolescente. BuenosAires, Argentina.

Coloma, C., 1991. Separata del curso desarrollo humano. PUCP. Lima.

Comisión Económica para América Latina y El Caribe (CEPAL). 1992. Tendencias actuales y perspectivas de los jóvenes de América Latina y El Caribe. División de Desarrollo Social. Santiago-Chile.

Chueca Ostola, A. 1994. Conocimiento de salud reproductiva en las gestantes adolescentes atendidas en el Hospital Nacional Cayetano Heredia. Lima.
Encuesta demográfica y de salud familiar 1999-2000. Informe Principal. Lima.

Encuesta demográfica y de salud familiar 1995-1996. Informe Principal. Lima.

Encuesta demográfica y de salud familiar 1991-1992. Informe Principal. Lima.

Gagnon, J., Parker, R. 1995. Concerving Sexuality: approches to sex research in a post - modern World. Routledge, New York and London.

Género y salud colectiva. 1997. VIII Congreso Latinoamericano de medicina social. Buenos Aires, Argentina.

Gogna, M., Panthelides, E., Ramos, S. 1996. Factores culturales y psicosociales en la prevención y tratamiento de las enfermedades de transmisión sexual: poder, placer y afecto en las negociaciones entre géneros. Informe de investigación. Buenos Aires, Argentina. 